\title{
Multiple-choice questions
}

\section{Multiple-choice questions in thoracic transplantation medicine}

\author{
S W Dubrey, C Melikian, N R Banner
}

Are the statements listed beneath each question true or false? Answers are given on pages 122-4.

\section{Question 1}

In the selection of donors and recipients for heart transplantation:

A HIV positive status in the recipient is an absolute contraindication

B Pulmonary hypertension in the donor is a relative contraindication

C Body size is an important consideration in matching donor and recipient

D Medical history of tuberculosis in the recipient is a contraindication

$\mathrm{E}$ Cytomegalovirus (CMV) positive status in the donor is a contraindication

\section{Question 2}

In transplant patients on immunosuppressive therapy, the following agents are correctly paired with their recognised potential side-effect profile:

A Azathioprine: nephrotoxicity, hypertension and neurotoxicity

B Corticosteroids: leucocytosis, affective disorders and osteoporosis

C Cyclosporin: bone marrow suppression, pancreatitis and hepatitis

D Antithymocyte globulin: anaphylaxis, leucopenia and haemolysis

E Mycophenolate: bone marrow suppression and haemorrhagic cystitis

\section{Question 3}

Immunosuppressed transplant recipients should not have the following:

A BCG vaccine

B Camembert cheese

C Varicella immune globulin

D Influenza vaccine

E Malaria prophylaxis

\section{Question 4}

The following drugs have important interactions with either cyclosporin or azathioprine

A Allopurinol

B Non-steroidal anti-inflammatory drugs (NSAIDs)

C Erythromycin

D Metformin

E Warfarin

\section{Question 5}

In the diagnosis of acute rejection of a transplanted organ:

A Cardiac rejection is usually accompanied by an increase in ECG voltage amplitude

B Obliterative bronchiolitis is characteristic of acute pulmonary rejection

C Cardiac rejection may cause left ventricular systolic dysfunction

D Rejection is excluded by the findings of a pyrexial patient with flu-like symptoms

E An asymptomatic patient may have significant histological rejection on cardiac biopsy

\section{Question 6}

In the selection of recipient candidates for thoracic organ transplantation:

A Irreversible pulmonary hypertension is a contraindication to orthotopic heart transplantation

B A prior history of hepatitis C is an absolute contraindication to transplantation

C Matching of body size is less critical for heart than for lung transplantation

$\mathrm{D}$ Insulin-dependent diabetes in the recipient is an absolute contraindication

E Creatinine clearance of $<50 \mathrm{ml} / \mathrm{min}$ and proteinuria of $>0.5 \mathrm{~g} / 24 \mathrm{~h}$ would prohibit transplantation

\section{Question 7}

In patients transplanted and on conventional immunosuppression:

A Pneumocystis carinii is the commonest cause of pneumonia in lung transplant patients

B A pre-transplant history of tuberculous lymphadenitis would not require prophylactic therapy

C Angina can never be appreciated in the heart transplant recipient due to denervation

D Coronary artery disease develops more rapidly in the transplanted heart than in a native heart

E Lymphoproliferative disease is frequently caused by CMV infection 


\section{Question 8}

In patients being assessed for transplantation:

A Patients with pulmonary histiocytosis $\mathrm{X}$ are at risk of recurrent disease after lung transplantation

B Amyloid heart disease is an absolute contraindication to cardiac transplantation

C Current cigarette smoking should be an absolute contraindication to thoracic organ transplantation

D A lack of social and domestic support is not a contraindication to transplantation

E Ventricular arrhythmia resistant to treatment with drugs or automatic implantable cardioverter defibrillators is an indication for heart transplantation

\section{Question 9}

In patients who have undergone thoracic organ transplantation:

A One year mortality for adult heart transplantation is $10 \%$

B Detection of aspergillus on broncho-alveolar lavage indicates invasive disease

C The dose and number of immunosuppressive agents may be reduced as time elapses following transplantation.

D The commonest indication for heart transplantation in adults is ischaemic cardiomyopathy

E The commonest indication for heart transplantation in children is congenital heart disease
Question 10

The following malignancies are a particular problem in patients on immunosuppressive therapy:

A Melanoma

B Non-Hodgkins lymphoma

C Sunlight-related skin malignancies.

D Kaposi's sarcoma

E Bronchogenic carcinoma 


\section{ANSWERS}

\section{Question 1}

A True

B False

C True

D False

E False

Absolute contraindications to the use of a donor heart for transplantation include coronary artery disease, previous myocardial infarction, ventricular arrhythmias, severe hypokinesis, HIV positive status, death due to carbon monoxide poisoning and metastatic cancer.

Relative contraindications include prolonged inotropic support, sepsis, prolonged hypotension, a long ischaemic time and a history of intravenous drug abuse. All potentially cause complications but the benefits must be weighed against the potential recipient's immediate prognosis. A history of tuberculosis in the recipient is not an absolute contraindication but it would have required appropriate treatment at the time and would necessitate isoniazid prophylaxis after transplantation. If a high-risk donor is proposed then it must be with the recipient patient's prior informed consent. CMV positive status is not a contraindication to transplantation in either the donor or recipient although reactivation of latent infections after immunosuppression may cause active infection.

\section{Question 2}

A False

B True

C False

D True

E False

Azathioprine, corticosteroids and cyclosporin are often used in maintenance immunosuppression. The major side-effects of cyclosporin are nephrotoxicity (acute and chronic), neurotoxicity (headaches, tremor and convulsions), hypertension and cosmetic problems (gum hyperplasia and hirsutism). These side-effects are shared by the newer immunosuppressant tacrolimus which can be used in place of cyclosporin.

Azathioprine may cause bone marrow suppression, pancreatitis, hepatitis and gastrointestinal upset. In the event of any of the above then cyclophosphamide may be a reasonable alternative agent. Antithymocyte globulin is commonly used as an immunosuppressive induction agent to cover the early postoperative period and occasionally to treat acute severe rejection episodes. It is produced by immunising rabbits or horses with human lymphoid cells. The most frequent side-effect of these preparations are chills, rigors, flu-like symptoms and less commonly serum sickness or anaphylaxis. Patients are hence often premedicated with paracetamol, antihistamines and corticosteroids. A major consideration is also the increased risk of infection, particularly CMV, with the use of such preparations. Mycophenolate can cause gastrointestinal sideeffects, including nausea and diarrhoea, in addition to bone marrow suppression. Haemorrhagic cystitis is a side-effect associated with cyclophosphamide.

\section{Question 3}

A True

B True

C False

D False

E False

Immunosuppressed patients in general should not receive live vaccines such as BCG, MMR, oral polio, oral typhoid or rubella vaccine. Inactivated vaccines, such as influenza, hepatitis A or $\mathrm{B}$, pneumococcal and adsorbed tetanus vaccine are permitted, although these are likely to be less effective than in an immunocompetent individual. Varicella immune globulin is used in immunosuppressed patients who have come into contact with chicken pox or varicella zoster cases. Patients are advised to avoid eating foods liable to be contaminated with listeria, such as soft ripened cheeses, for example Brie, Camembert, blue-veined cheeses and all pates.

\section{Question 4}
A True
B True
C True
D False
E False

Many drugs interact with the standard immunosuppressive agents used. Cyclosporin is nephrotoxic and therefore combinations which increase the risk of nephrotoxicity, eg, NSAIDs, aminoglycoside antibiotics and vancomycin should be avoided. Allopurinol can only be used with careful monitoring as it inhibits the metabolism of azathioprine (usually a reduction in dose of azathioprine to $1 / 4$ the original dose is required). The combination can cause dangerous bone marrow suppression. Metformin is excreted via the kidneys and should be avoided when renal function is impaired. In addition, metformin has been shown to interact with contrast agents used in angiography, a procedure often performed annually in the follow-up of heart transplant patients. Thus, whilst not contraindicated in transplantation or with immunosuppressive medications per se, metformin should be used with caution when radiographic contrast agents are used.

\section{Question 5}

A False

B False

C True

D False

E True

Fever in a transplant recipient will most often be due to infection. A minority of patients with rejection can present with fever and generalised flu-like symptoms, reflecting the presence of an 'inflammatory' process. Most sinister is the presence of significant rejection with few or no symptoms and therefore surveillance right ven- 
tricular endocardial biopsy is utilised. Cardiac rejection is indicated by symptoms of dyspnoea, palpitations or loss of appetite and signs of heart failure including dependent oedema, a third heart sound and raised jugular venous pressure. The ECG will often show a reduction in voltage with significant rejection. In acute rejection diastolic dysfunction of the left ventricle appears to occur early; once systolic impairment is evident then urgent therapy is required. Echocardiography will usually show a global reduction in ventricular function; a regional abnormality being more suggestive of ischaemic damage from graft vascular disease (chronic rejection). Pulmonary rejection is indicated by symptoms of dyspnoea and by reduction in both air flow and lung volume on spirometry. Possible rejection is investigated by bronchoscopy, broncho-alveolar lavage and transbronchial biopsy to exclude infection as a differential diagnosis. Rejection can often be confirmed histologically from the transbronchial biopsy. Obliterative bronchiolitis is a feature of chronic rejection and not usually seen acutely or in the immediate time period after transplantation.

\section{Question 6}
A True
B False
C False
D False
E True

Whilst irreversible pulmonary hypertension is a contraindication to conventional orthotopic heart transplantation, the problem can be tackled by the use of a 'pressure primed' heart from a donor with primary pulmonary hypertension. Patients with cystic fibrosis who receive a heart and lung block may also donate their heart in a 'domino' donor programme to a recipient with pulmonary hypertension. A second procedure occasionally used is that of heterotopic transplantation, in which the donor heart is implanted in the right side of the chest, attached in parallel to the patient's native heart and vascular circuit, to act in effect as a physiologic assist mechanism. In this way the donor heart, with right ventricle attached via the pulmonary artery to the native heart's right atrium, or superior vena cava, is not exposed to the high pulmonary artery pressures.

The majority of individuals infected with hepatitis $C$ have persistent infection which may be aggravated by immunosuppressant drug therapy. Patients with pre-existing liver dysfunction or cirrhosis would be excluded but in other cases transplantation could be possible, albeit at an increased risk. Body size is paramount to the selection and matching of recipients to donor organs for both hearts, lungs and heart lung transplants. In the case of hearts, a $20 \%$ mismatch on body size is the maximum advisable. Insulin-dependent diabetes is a systemic disease but provided there is not significant end-organ damage (retinopathy, peripheral or cerebral vascular disease and renal impairment particularly), it does not represent an absolute contraindica- tion. Evidence of significant renal impairment (creatinine clearance $<50 \mathrm{ml} / \mathrm{min}$ ), of whatever aetiology, is a serious consideration usually resulting in the rejection of a candidate. Sometimes the renal dysfunction can be secondary to heart failure and may be improved with inotrope therapy or mechanical circulatory support prior to transplantation. The consideration is that with the obligatory introduction of cyclosporin and the peri-operative stress there will be further reduction in renal performance that could result in the need for dialysis.

\section{Question 7}

A False

B False

C False

D True

E False

The commonest opportunistic infection in both heart and lung transplant patients is CMV infection. This usually responds to treatment with intravenous ganciclovir. Tuberculosis may be reactivated by steroids and chronic immunosuppressive therapy. Angina may be appreciated in heart transplant patients several years after their surgery; the mechanism presumably due to re-innervation of the donor heart. Lymphoproliferative disease resulting from immunosuppressive therapy is associated with the Epstein Barr virus in most cases and will frequently respond to a reduction in immunosuppression and/or treatment with aciclovir.

\section{Question 8}

A True

B False

C True

D False

E True

Amyloid heart disease is usually part of a systemic process and recurrence of amyloid deposition in the transplanted organ is well documented. However, if the primary disease process of amyloidosis is also treated then heart transplantation should not be considered an absolute contraindication. Continued cigarette smoking should be considered an absolute contraindication, particularly with the added risk of accelerated coronary artery disease. Social, domestic and family support are essential components to recovery from organ transplantation; these components form an essential part of a potential transplant recipients evaluation.

\section{Question 9}

A False

B False

C True

D False

E True

Current estimates from the International Society of Heart \& Lung Transplantation show a one-year mortality for heart transplantation of $20 \%$, with an approximate $4 \%$ mortality per year thereafter. Isolation of Aspergillus from pulmonary secretions does not necessarily 
imply invasive disease. The mainstay of diagnosis of invasive aspergillosis is its detection in tissue where branched septate hyphae are seen on histology of lung biopsies. The commonest indication for heart transplantation in adults is currently dilated cardiomyopathy of nonischaemic origin.

\section{Question 10}
A False
B True
C True
D False
E False

An unfortunate consequence of chronic immunosuppression is an increased risk of a number of malignancies due to a loss of T-cell immune surveillance. In general, the risk of malignancy is three times that of an age-matched nontransplant population; some malignancies are particularly common. Lymphoproliferative tumours, often in younger recipients, are perhaps the most important and can occur early after transplantation. As the risk of most malignancies increases with age and the addition of immunosuppressive therapy may accelerate the progression of occult malignancy, age and/or symptom driven screening tests should be performed in all candidates for transplantation.

\section{Images in medicine}

\section{Skull osteomyelitis and multiple brain abscesses}

Figure 1 Skull X-ray
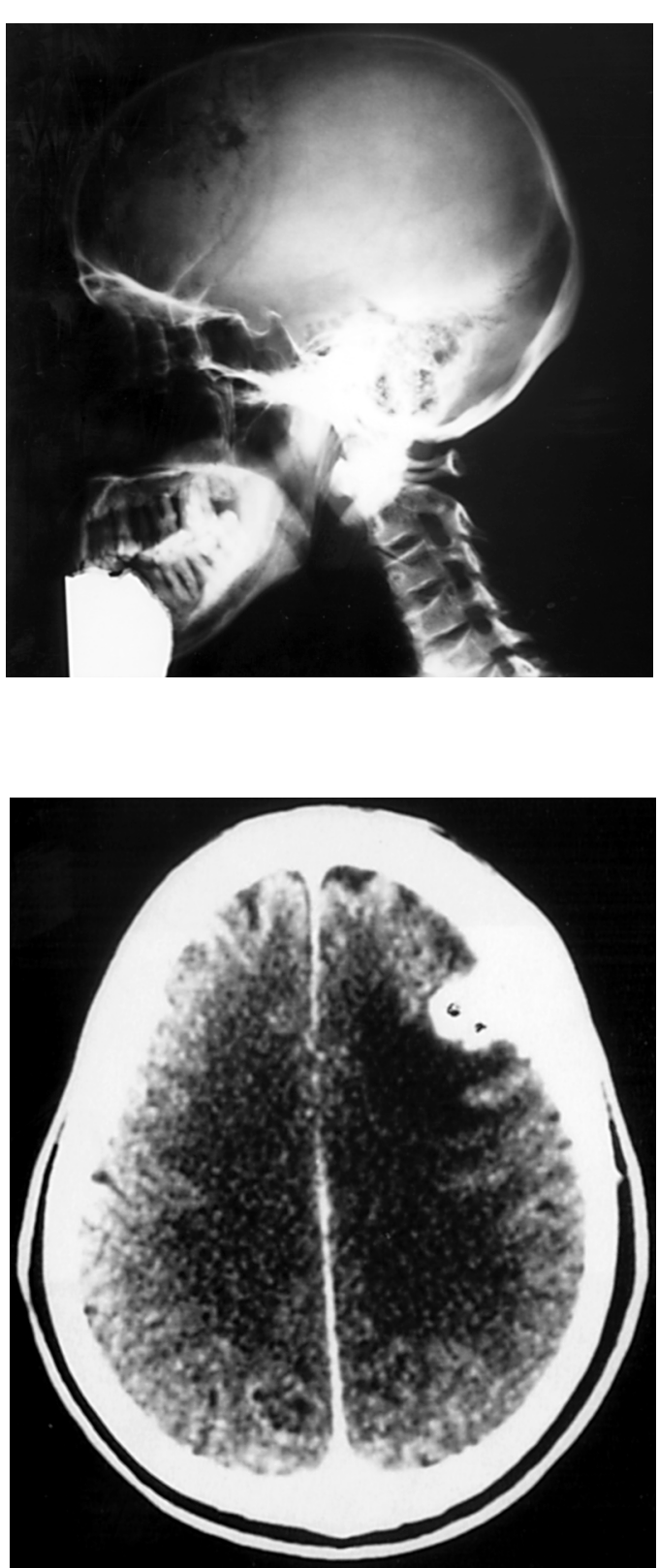

A 52-year-old diabetic Indian man presented with a 2-week history of headache and fever. $\mathrm{He}$ had no evidence of meningism. There was mild polymorph leucocytosis and raised erythrocyte sedimentation rate of $113 \mathrm{~mm} / \mathrm{h}$. Other investigations, including tests for human immunodeficiency virus and immunoglobulins, yielded negative or normal results. Initial skull X-ray and brain computed tomography (CT) scan were normal. Three days later, he was found to have subcutaneous swelling over the left frontoparietal region of the scalp. Incision drainage revealed a subaponeurotic abscess in direct contact with the bone. Pseudomonas cepacia was grown from pus and treated with appropriate antibiotics. Fever and headache settled and he became asymptomatic after few days. Two weeks later, a discharging sinus with serosanguinous material from the scalp wound was noted. At this point, osteomyelitis of the cranial vault was clearly seen on plain skull X-ray (figure 1). A follow-up brain CT scan showed left-sided soft tissue swelling and multiple ring-enhancing frontal lobe abscesses (figure 2). Surprisingly, apart from the scalp discharge, he remained asymptomatic. $P$ cepacia was again grown from the wound and treated with a combination of piperacillin and ceftazidime. Three weeks later, a repeat CT scan showed marked improvement of his intracerebral and scalp lesions. The antibiotics were continued for another 3 weeks. This case demonstrates that even a minor superficial suppurative lesion has the potential to become sinister in an immunocompromised subject, as it may rapidly extend to deeper tissues. Moreover, the diagnosis of these multiple and apparently silent cerebral abscesses would have been delayed if repeated radiological imaging had not been undertaken.

HAIDER M AL ATTIA RAHEEL A QURESHI IDRIS A EL HAG 